18

\title{
Magnetic Circular Dichroism in 2D Colloidal Semiconductor Nanocrystals*
}

(C) Y.A. Gromova, M.A. Miropoltsev, S.A. Cherevkov, V.G. Maslov, A.V. Baranov, and A.V. Fedorov

ITMO University,

197101 St. Petersburg, Russia

e-mail: yulia.a.gromova@gmail.com

Received July 6, 2018

Magnetic circular dichroism (MCD) spectra were measured for colloidal CdSe/CdS core-wing nanoplatelets (NPLs). MCD spectra of CdSe cores demonstrate well resolved features which could be attributed to excitonic transitions from heavy hole, light hole and split-off sublevels. A1/D0, B0/D0 MCD characteristic terms were determined. The values of A1/D0, B0/D0 terms have no dependence on NPL thickness and are very close to the corresponding values in organic molecules.

DOI: $10.21883 /$ OS.2018.11.46827.218-18

\footnotetext{
* International Conference „PCNSPA 2018 - Photonic Colloidal Nanostructures: Synthesis, Properties, and Applications“", Saint Petersburg, Russia, June 4-8, 2018.

Полный текст статьи опубликован в английской версии журнала.
} 